\title{
Experts' Judgment-Based Mamdani-Type Decision System for Risk Assessment
}

\author{
Fatin Amirah Ahmad Shukri (iD ${ }^{1,2}$ and Zaidi Isa ${ }^{1,3}$ \\ ${ }^{1}$ School of Mathematical Sciences, Faculty of Science and Technology, National University of Malaysia, Bangi 43600, \\ Selangor, Malaysia \\ ${ }^{2}$ Centre for Foundation Studies, National Defense University of Malaysia, Sg Besi Camp, Kuala Lumpur 57000, Malaysia \\ ${ }^{3}$ Risk Management Centre, Sustainable and Occupational Health (ROSH-UKM), National University of Malaysia, \\ Bangi, Malaysia
}

Correspondence should be addressed to Fatin Amirah Ahmad Shukri; fatin@upnm.edu.my

Received 6 December 2020; Revised 26 June 2021; Accepted 20 September 2021; Published 2 November 2021

Academic Editor: Luigi Rodino

Copyright (c) 2021 Fatin Amirah Ahmad Shukri and Zaidi Isa. This is an open access article distributed under the Creative Commons Attribution License, which permits unrestricted use, distribution, and reproduction in any medium, provided the original work is properly cited.

\begin{abstract}
Mamdani fuzzy inference system has been widely used for potential risk modelling and management. The decision-making is usually provided by multiple experts in the field. The conflicting information in sources from different experts become an open issue and has attracted some researchers to investigate further. Various risk factors in a project caused difficulties for decision makers to make reliable decisions on the whole project since it involves ambiguities, vagueness, and fuzziness. The introduction of the fuzzy inference system to the evaluation of construction risk is capable in explaining its reasoning process and, hence, overcoming such problems. Risk factors under the project management risk were identified through literature sources and from the opinion of experts. It is found that the likelihood and severity of risk is somehow interlinked with the concept of fuzzy theory. For model input and output linguistics variables, the triangular membership function was selected. The methodology employs a fuzzy aggregation system in which an appropriate control action can be determined by the acquisition of expert judgment. A total of 23 rules with logical OR operator, truncation implication, and Mean of Maxima (MoM) method for defuzzification were used to create an effective fuzzy model intended for making decisions. The framework determines the relationship between input and output parameters in if-then rules or mathematical functions using an effective fuzzy arithmetic operator. The study addresses the principle issues of multiexpert opinions based on Mamdani-type decision system and the illustrative example taken from one of medium-sized project held in Malaysia's construction industry. By comparing with other experimental results, we verify the rationality and reliability of the proposed method.
\end{abstract}

\section{Introduction}

The construction industry is highly associated to risks among other industrial sectors, due to the nature of its business on construction activities namely, financial strength, long-term mode of business, environmental complexity, and vigorous structure of an organization [1]. Project constructions bare risks that undeniably affect the performance related to budget, project objectives, and schedule. Therefore, the risks of the project must be identified and quantified before the project begins and finally provide adequate measures in order to mitigate the risks. The definition of risk arbitrarily varies according to many aspects. Risk is generally defined as the probability of loss $[2,3]$ and damage caused to an event that might affect the objectives and projects in the short as well as long term. Therefore, effective risk management in construction projects is critical and, hence, need greater attention. Monitoring and analysis of risks are part of the process [4]. There are many approaches used by those involved in risk management to assess the risk starting from an easy approach to a more complicated method. It requires more time and financial investment in the context of a comprehensive analysis of the risks that may ultimately affect a specific 
project, and in many cases, it is clearly not sufficient to cover the risks that occur as a result of the implementation of the project.

As the primary concern of this paper lies on the multiexpert judgment in risk assessment, it is therefore vital to define the correct form of risk analysis. Analogously, human decisions are made during cognitive and behavioural activities involving ambiguity and subjective attributes [5]. Expert knowledge is a viable informative data due to the uncertainty and imprecision for problematic risk analysis limitations [6]. The assessment of risk depends on the organizational objectives and has two main approaches, i.e., qualitative and quantitative approach. Quantitative analysis uses statistical methods and models to build probabilistic loss estimates, which simulate the entire project where the time and total costs of each of the critical risks will be studied. On the contrary, the qualitative method uses the rating event impact and event likelihood on a Likert scale (low, medium, and high) and uses a risk matrix to determine the risk result. A basic risk calculation is Risk $=$ Probability $\times$ Loss. When evaluating the qualitative risk, the probability of a given occurrence and its effects are measured or estimated, and then, the empirical parameters are used to determine the acceptability of the risks. The use of fuzzy logic (FL) in decision-making has increased significantly in recent years and is seen as a new and efficient tool in risk assessment. Furthermore, the basis of FL is easy to understand which is expressed in natural language, flexible, and can deal with imprecise data without necessarily acquiring mathematical models of the system to be controlled.

Construction risk management and assessment is very complicated owing to various factors. The maximum number of criteria will be taken into consideration in order to correctly measure the probability and impact of a project risk. In order to respond to various ranges of risks, there must be an established and objective risk management practice. The trend in Malaysia's construction industry has shown a lack in risk management practice [7] among industry practitioners, especially on the quantitative or qualitative measures being applied in the small- and medium-sized construction project. In addition, the industry players themselves reluctantly implemented risk management tools due to lack of knowledge in this field and financial constraint of its organization [8]. There is a need for a large amount of investment by construction companies towards risk management, as the risk and uncertainty in project construction to a certain degree is unbearable and intolerable over time. To overcome these uncertainties, many efforts have been taken to introduce expert systems and sets for effective decision-making in any processes.

This research is motivated by two factors. First, it should be emphasized that there is a lack of quantitative discipline in the literature on systematic risk evaluation and the design of acceptable risk response for construction projects. The methodology suggested in this study is anticipated to help managers acquire a better understanding of key high-risk areas that demand immediate attention. Secondly, there have been few past studies in Malaysia's construction sector that have validated the risks and the usage of professional guidance on risk assessment. Generally, an expert is someone who has conducted specialized study or has knowledge in academic, technological, or other fields. Experts' opinions are frequently used as a reference in a subject. However, since various experts have diverse backgrounds, their evaluation of the same event is frequently subjective and ambiguous. This makes our practical application more challenging. With the presence of imprecise and partial data information, a fuzzy inference system (FIS) is often developed [9]. Moreover, it is commonly gained through the use of expert opinion. A broad FIS application in a variety of risk assessment areas is accountable in the construction risk evaluation, leading to the use of fuzzy reasoning. Moreover, it is generally obtained using the knowledge of experts' opinion. A broad FIS application in numerous risk assessment areas is liable in the construction risk evaluation, thus leading to the usage of fuzzy reasoning [10]. This study proposes a better analytical technique based on triangular fuzzy numbers and modifying the membership functions and inference procedures. The insights acquired from implementing this proposed strategy to a medium-range project construction industry that draws practitioners' attention to critical areas of improvement. This is designed to promote the development of greater understanding among decision-makers in their respective operational domains.

The key contributions of the suggested approach, in comparison to other existing fuzzy logic methods, are as follows: we utilize triangular fuzzy numbers for the evaluation results of various experts, and we use combined risk assessment results obtained from the aggregation of all risk inputs. The traditional fuzzy system techniques deal with dense rule bases, in which the universe of discourse is completely covered by the antecedent fuzzy sets of the rule base in each dimension, resulting in at least one activated rule for each input. The primary issue with these traditional methods is their considerable computational complexity [11]. Due to the limited number of input variables, Mamdani's fuzzy reasoning model is utilized and therefore the minimal feasible number of reasoning rules. If there are differing viewpoints, more explanation from experts may be required to comprehend the reasoning. In other words, changes to the output membership function are permitted. A suggested risk model with specified variables, membership functions, and inference rules will be conveyed back to experts for feedback and approval. The suggested technique has the benefit of being theoretically applicable to all forms of risk assessment utilizing numerical approaches, particularly when risk-related data are difficult to collect or scarce.

This paper focuses on the development of a Mamdanitype fuzzy inference process in construction risk assessment that could be applied by the decision makers. Four risk factors were considered for the development of the risk assessment model and the ratings of each risk item were evaluated by four experts in this field. Section 2 addresses the relevant works in previous risk assessment literature, followed by a brief overview in fuzzy logic in Section 3. Section 4 highlights the detailed step-by-step process of the fuzzy decision system, and Section 5 presents the numerical example of the subject under the study. Section 6 then concludes the proposed method. 


\section{Related Work in the Risk Assessment Method}

Numerous methodologies for risk assessment have been proposed in various fields. Risk assessment of the project is, therefore, very relevant, and much work has been done for project evaluation. The construction site is usually preoccupied, and hence, the activities of a building development in urban areas are difficult to supervise. Forbes et al. [12] developed a matrix for selecting appropriate risk management techniques in the built environment for each stage of risk management. These techniques include artificial intelligence, decomposition, probabilistic analysis, sensitivity analysis, and decision trees, among others. The research proposed a quantitative risk assessment (QRA) method of risk management in the urban area sector. Later, an improved QRA model to quantify risks for nonhomogenous urban growth was designed, since the current QRA models for commercial construction are irrelevant, which was proposed by Meng et al. [16]. Many authors used the probability method for construction risk in the last decade such as Monte Carlo [1], event tree analysis [6, 14, 15], failure mode and effect analysis [16], risk matrix [17], and multicriteria decision-making [1].

Recent trends have shown that authors prefer soft computing in risk assessment. Yazdani et al. [18] developed a fuzzy risk assessment model to assess risk and management for electricity distribution system asset protection. The research was conducted using a comparison analysis to depict the effectiveness and capability of the new risk assessment model. In 2017, Fayaz, Ullah, Park, Kim, and Kim introduced a hierarchical fuzzy inference-based model for underground risk assessment. The research proposed new rules, namely, maximum rules and average rules, which are interchangeably applicable according to the required rationale for rule design. This greatly decreases the curse of dimensionality. The value of the fuzzy model [4] later discussed the ability to convert the input variables, the number of subrisks (NSR) and the total value of subrisks (TVSR), into linguistic variables and evaluation, total value of project risk (TVPR) of the output variable. Using this method, the risk factor and volatility that is often correlated to real programs can be simulated. A case study discusses the scheme of the model, the rule block, the characteristics, and the membership functions. In the process of making decisions where classification by algorithms is incredibly difficult and parameters are compounded, the use of fuzzy logic is an advantage.

The research by Gupta and Thakkar [1] adopts an adaptive risk prioritization method using team strategy for order preference by similarity to ideal solution (GTOPSIS) and quantifies consequences for cumulative project delays using the judgmental risk assessment procedure (JRAP) and Monte Carlo simulation (MCS) methodology. The findings of the qualitative risk analysis by GTOPSIS were compared with the quantitative risk analysis, i.e., JRAP and MCS. Kim et al. [19] suggested a quantitative risk assessment approach for construction sites using risk indicators to predict economic damages. The study used multiple regression approach to identify risk factors, using the payment of a construction site compensation insurance firm to represent the actual financial losses in South Korea as an illustrative example. The outcome of this research was accepted as a needed guideline for developing a construction risk estimation model. The recent study on risk management field, by Barghi and Shadrokh Sikari [20], lists 32 risk factors, and their number reduced to 17 risks using the expert opinion via fuzzy Delphi technique after reviewing the literature and the project management body of knowledge (PMBOK). Three levels of study are executed. The findings of the confirmatory factor analysis revealed that the research participants accepted all risks. Based on the literature review, it was observed that there is still a lack of research on decision-making based on expert judgment, especially in the Malaysia construction industry context.

\section{Fuzzy Sets Theory}

Zadeh [21] had initiated fuzzy sets and fuzzy logic (FL). FL is a type of soft computing system that accommodates realworld imprecision. Specifically, FL is an extension of multiassessed logic, the purpose of which is to estimate reasoning rather than providing exact solutions. The conventional binary logic (crisp logic) way displays either 1 or 0 representing truth or false, respectively, whereas the degree between 0 and 1 in FL variables may have truth values within the range [22]. Instead of describing the absolute yes or no, the membership function (MF) truth values are explained in terms of degree. Fuzzy sets are generated for each input and output variables by dividing the discourse universe into various subregions, referred to as linguistic variables. A linguistic variable is a variable whose values are represented in phrases or words in natural language and defined by an acceptable MF [17]. Specifically, MF is a curve that determines how the membership value between 0 and 1 maps every point in the input field. Here, 0 signifies absolutely false, while 1 signifies absolutely true, and the degree of trueness is within the specified range. Modelling uncertainties, vagueness, and subjective attributes require more than probability theory knowledge. Arithmetically, an element $x$ belonging to a set $A$ is defined as $x \in A$, and an element that is not a member in $A$ is denoted as $x \notin A$. The definition of characteristics function or membership function, $\mu_{A}(x)$, is an entity that has a crisp value of 1 or 0 in universe $\mathrm{U}$. For every $x \in \mathrm{U}$,

$$
\mu_{A}(x)= \begin{cases}1 & \text { for } x \in A, \\ 0 & \text { for } x \notin A,\end{cases}
$$

which can also be expressed as $\mu_{A}(x) \in\{0,1\}$.

For the classical set or crisp set, the MF will take a value of 1 or 0 , while for fuzzy sets, the MF may take values in the interval of $[0,1]$. The range between 0 and 1 is referred to as the membership grade or degree of membership [23]. A fuzzy set $A$ is defined as follows:

$$
A=\left\{\left(x, \mu_{A}(x)\right) \mid x \in A, \mu_{A}(x) \in[0,1]\right\},
$$

where $\mu_{A}(x)$ is a membership function in between the interval of 0 and 1 . The union and intersection are two 
operations commonly used in a set theory. The union and intersection of set $A$ and set $B$ can be defined as

$$
\mu_{A \cup B}=\max \left\{\mu_{A}, \mu_{B}\right\}
$$

and

$$
\mu_{A \cap B}=\min \left\{\mu_{A}, \mu_{B}\right\}
$$

\section{Fuzzy Inference System}

A fuzzy inference system (FIS) is a system that applies fuzzy set theory to map inputs to outputs. The calculation of fuzzy logic consists of three steps given by fuzzification, fuzzy inference, and defuzzification, as shown in Figure 1.

The fuzzification implies that the real variables are transferred on linguistic variables [24]. Crisps and real values are changed to linguistics function and fuzzy sets in fuzzification. Then, the membership function is set up for input and output variables (Figure 2). The fuzzy inference determines the actions of the system by the laws of $<$ If $>$ and $<$ Then $>$ at the linguistic stage where the conditional clauses determine the position of the input variables by the laws. The conditional clauses are in the form of

$$
<\text { If }>\text { Input } a<\text { And }>\text { Input } b, \ldots \text {, Input } x<\text { Or }>\text { Input } y, \ldots \ldots,<\text { Then }>\text { Output } 1 \text {, }
$$

which implies that when "the state occurs," it is simply called antecedent Input a and Input $b, \ldots .$. . Input $x$ or Input $y, \ldots . .$. ; then, "the situation is" is called consequent Output1.

The fuzzy logic depicts the system of expert. The combination of variables attributes inside the structure and occurring in $<$ If $>$ and $<$ Then $>$ condition represents one rule. Every $<$ If $>$ condition has the corresponding consequence for $<$ Then $>$. Each rule must be calculated with its level of support (the weight of rule in the system) and the experts will form the rules. The linguistic terms and membership function are defined to determine the degree of truth for each assumption, based on the actual and real values. The defuzzification moves the effects of the fuzzy inference to the output variables that verbally explain the outcomes (for example, whether there is a risk or not). The fuzzy logic system will operate automatically with input data entered, in which the input data can be interpreted by a variety of variables.

4.1. Mamdani-Type Fuzzy Inference Process. There are generally two types of fuzzy inference methods, i.e., Mamdani and Sugeno fuzzy inference methods. Mamdani fuzzy inference is the most commonly used inference method in application due to its simple structure of minmax operations. Mamdani and Assilian [25] have implemented this approach. Another well-known method of inference is the Sugeno or Takagi-Sugeno-Kang (TSK) method of fuzzy inference proposed by Takagi and Sugeno [26]. Consequently, the two methods can be executed into two procedures.

The first step is the crisp value fuzzification of input variables with the correct fuzzy sets into the membership values, where the process for both methods is precisely the same. The main difference between the two methods lies in the consequent of fuzzy rules. Although variations exist in the second method, the effects of all the rules are combined into a single, valid performance output value. Through Mamdani inference, a fuzzy set determines the outcome of the if-then rule [27]. A matching number will be reworked in the output fuzzy set of every rule and defuzzification after the accumulation of all the reshaped fuzzy set is performed. Therefore, in Sugeno's inference, a polynomial in relation to the input variables is the result of the if-then rule, which implies that each rule is provided with one number. The final crisp output is then carried out by using a weighting mechanism. Although Sugeno's inference prevents complicated defuzzification, the task of evaluating polynomial parameters is inefficient and less simple than specifying the fuzzy output sets for Mamdani inference [28]. The current paper discusses the method using Mamdani inference.

4.2. Basic Steps in FIS. The steps of fuzzy logic (inference operations upon fuzzy if-then rules) conducted by FISs are as follows:

Step 1: fuzzification compares the input variables to the membership functions in the following section in order to achieve the membership values of each linguistic label.

Step 2: fuzzy inference combines the fuzzified inputs according to the fuzzy rules to establish a rule strength (fuzzy operations).

Step 3: it finds the outcome of the rule by combining the output membership function and the rule strength (implication).

Step 4: defuzzification aggregates the qualified consequents to produce a crisp output using Mean of Maxima (MoM) defuzzification operator [29]:

$$
Z=\frac{\sum_{i=1}^{n} w_{i} x_{i}}{\sum_{i=1}^{n} x_{i}},
$$

where $Z$ is the defuzzified output, $\mu_{i}(x)$ is the aggregated membership function, and $x_{i}$ is the support value at which the $i^{\text {th }}$ membership function reaches its maximum value.

The study adopts one of the widely used method for defuzzification, i.e., the mean of maximum (MoM). By using the MoM method, the observation results were 


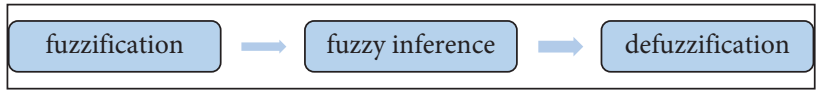

Figure 1: Decision-making by FL.

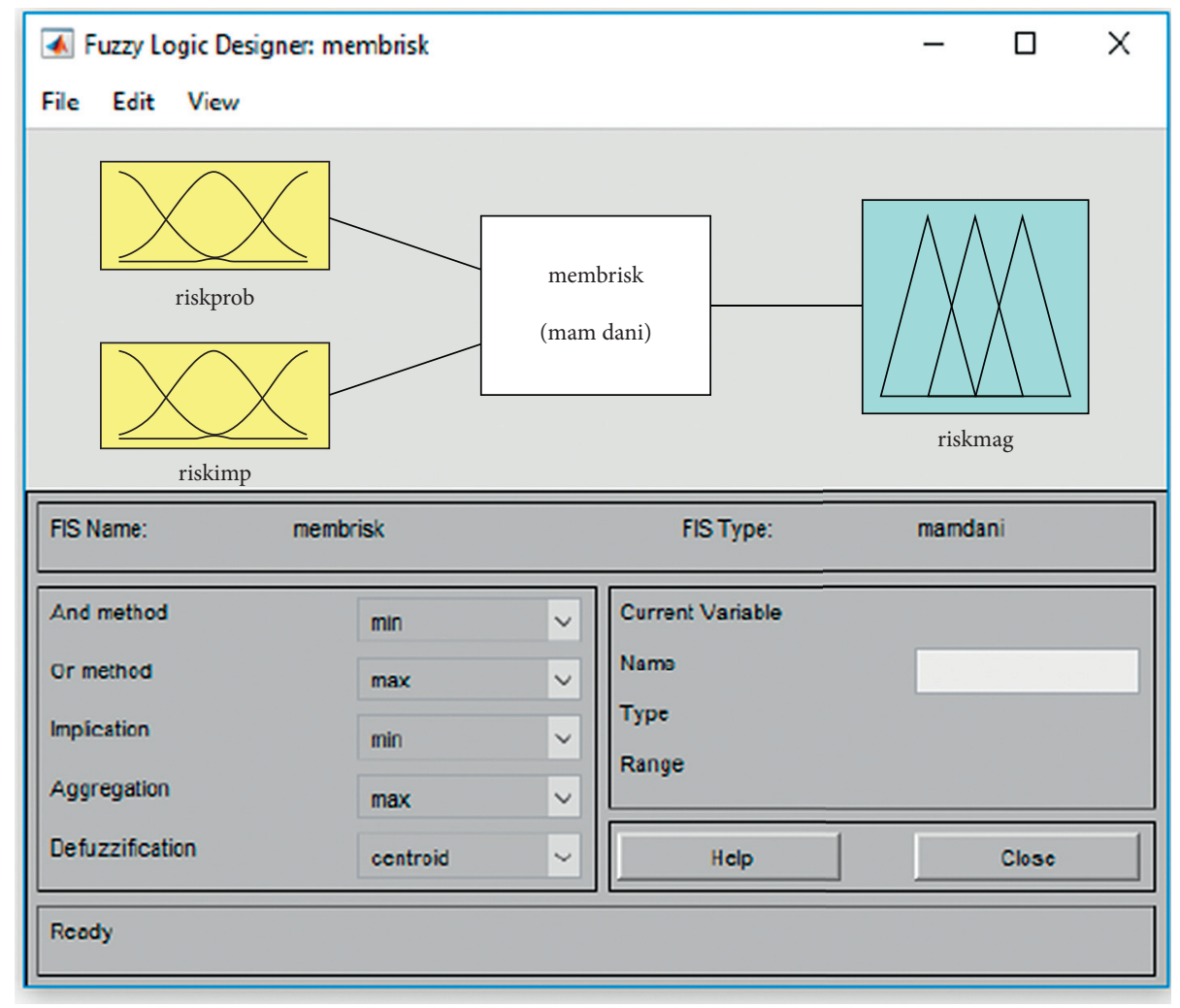

Figure 2: Fuzzy model.

computationally simpler yet obtained accurate results. Figure 3 displays the use of if-then type fuzzy rules which converts the fuzzy input to the fuzzy output.

\section{Numerical Example}

A numerical example of "Project Management Risk" is designed to explain the full process of fuzzy inference by Mamdani. Experts who have extensive experience in construction projects (consists of civil engineer, architect, quantity surveyor, and project manager) involved in a medium-sized project were selected to answer a questionnaire containing items obtained through the analysis of the findings from the risk likelihood (RL) and the risk severity (RS), items with reference by selected papers [30]. As agreed, the likelihood of RL and RS was selected as the inputs for assessing the criteria of risk (RC) for the risk items: processes $(r 1)$, resources $(r 2)$, inexperience team $(r 3)$, and lack of management or control $(r 4)$. To make it simpler, the 5 levels of risk likelihood, 5 levels of risk severity, and 5 levels of risk criteria are defined in Table 1. The degree of likelihood is demonstrated into five different linguistic variables: improbable, remote, occasional, probable, and frequent. Whereas the five different linguistic variables for degree of severity are negligible, minor, serious, critical, and catastrophic. The following table shows the levels of linguistic variables for the three inputs with the corresponding fuzzy numbers.

The fuzzy system was implemented in MATLAB using the following properties: defuzzification = "MoM" (Mean of Maximum), decision method for fuzzy logic operators OR = "MAX," type = "mamdani," implication method = "MIN," and aggregation method = "MAX." After the implementation of the program in MATLAB, the validation dataset was inserted into the model and each risk object was categorized as Tolerable (T), Low (L), Medium (M), High (H), and Intolerable (IN). The most common approach for measuring the overall performance of risk is to use numerical numbers according to their achievement in respective criterion. Unfortunately, this method often results in fixed number and ignoring the vagueness in the system. From this point of view, the work is proposed to measure using fuzzy logic.

\section{Mamdani-Type Decision System}

Firstly, we fuzzified all the crisp inputs and classify the degree of the related fuzzy sets of which the inputs 


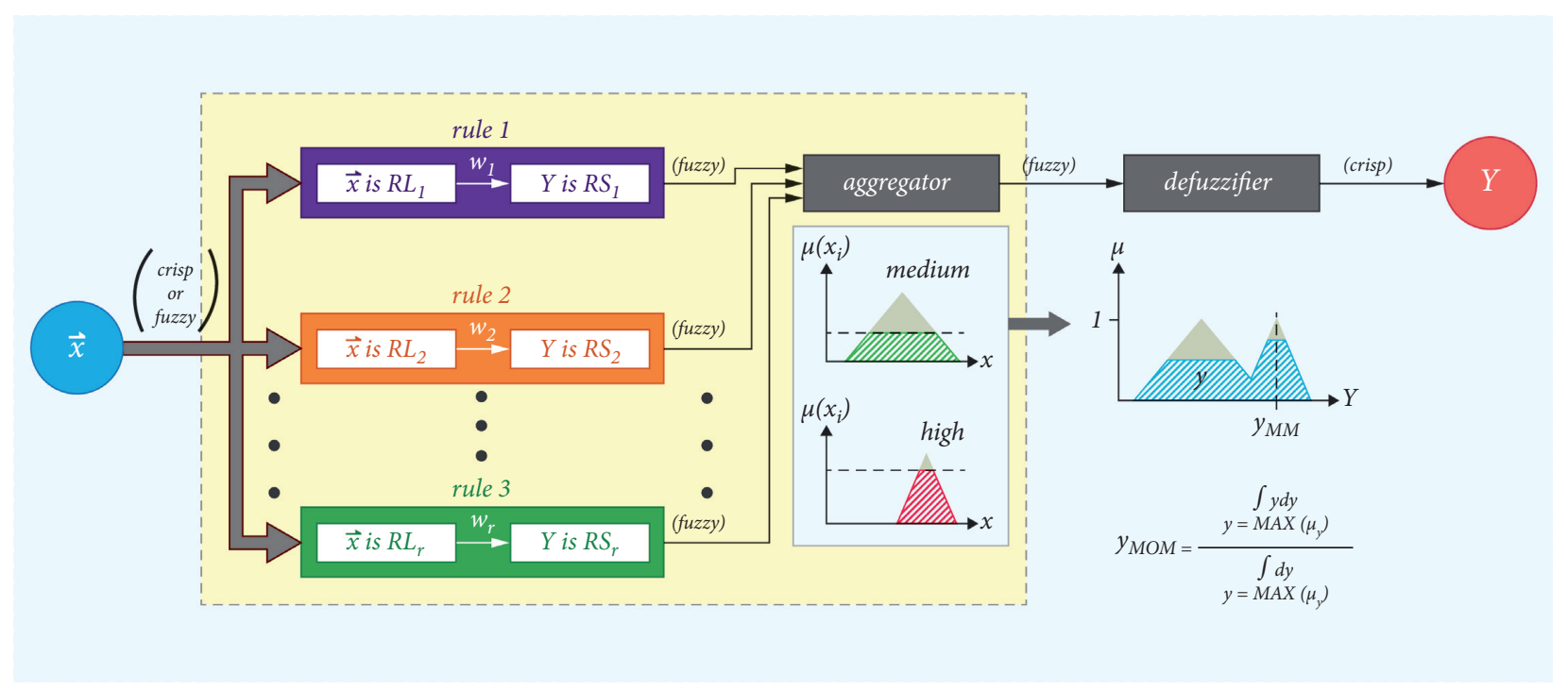

Figure 3: Fuzzy if-then rule.

TABLe 1: Fuzzy sets for risk likelihood and risk severity.

\begin{tabular}{|c|c|c|c|}
\hline Variable & Parameter & Description & Fuzzy numbers \\
\hline \multirow{5}{*}{ Risk likelihood } & Improbable (I) & Probability of risk to occur is very low & $(0,0,3)$ \\
\hline & Remote (R) & Probability to occur is relatively low & $(1,3,5)$ \\
\hline & Occasional (O) & Sometimes & $(3,5,7)$ \\
\hline & Probable $(\mathrm{P})$ & High & $(5,7,9)$ \\
\hline & Frequent $(\mathrm{F})$ & Probability to occur is almost certain & $(7,10,10)$ \\
\hline \multirow{5}{*}{ Risk severity } & Negligible $(\mathrm{N})$ & There is no delay or damage occured & $(0,0,3)$ \\
\hline & Minor (M) & Delay is relatively low and less damaged & $(1,3,5)$ \\
\hline & Serious (S) & Delay/damage occurred & $(3,5,7)$ \\
\hline & Critical (C) & Major delay and damage occurred & $(5,7,9)$ \\
\hline & Catasthropic (Ca) & Project failure & $(7,10,10)$ \\
\hline \multirow{5}{*}{ Risk criteria } & Tolerable $(\mathrm{T})$ & Insignificant risk & $(0,0,3)$ \\
\hline & Low $(\mathrm{L})$ & Risk is acceptable & $(1,3,5)$ \\
\hline & Medium (M) & Risk is moderately acceptable & $(3,5,7)$ \\
\hline & High $(\mathrm{H})$ & Risk is significant & $(5,7,9)$ \\
\hline & Intolerable (IN) & Risk is not acceptable & $(7,10,10)$ \\
\hline
\end{tabular}

RL, RS, and the underlying output RC are interpreted in the triangular MFS, as shown in Figure 4 as adopted from Carr and Tah [31], where Improbable $(\mathrm{I})=(0,0,3)$, Remote $(R)=(1,3,5)$, Occasional $(\mathrm{O})=(3,5,7)$, Probable $(\mathrm{P})=(5,7,9)$, and Frequent $(\mathrm{F})=(7,10,10)$. On the contrary, experts also provide the numerical scale of RC in percentages.

belong. Table 2 shows the expert's rating and its underlying fuzzy sets. The four experts are represented by E1, E2, E3, and E4. According to Table 2, the crisps' input $R L$ (risk likelihood rated by $\mathrm{E} 1$ as 8 out 10 in a linguistic scale) corresponds to the fuzzy membership of $R L_{i}$ and $R L_{j}$ (Probable and Frequent), respectively. Likewise, the crisp input $R S$ (rated as 7 by E3) corresponds to the degree of critical. While E1 gave estimation of the severity of certain risk as 8 which corresponds to the fuzzy membership of $R S_{i}$ and $R S_{j}$ (Critical and Catasthropic), respectively. The rule evaluation involved in the application of the fuzzified inputs to antecedents in the fuzzy rules. Therefore, we used the MIN operator to evaluate the fuzzy OR operation and the MAX operator to evaluate the fuzzy AND operation. The overall results are presented in Table 2.
6.1. Fuzzification. Experts' rating and its underlying fuzzy sets are provided in Table 2.

\subsection{Fuzzy Aggregation}

6.2.1. Unification of All Output of All Rules. In this regard, we used the clipped or scaled consequent MFS, meaning that we take intersections of the parameters between TFNs and MF. Once the crisp inputs were converted to its corresponding fuzzy sets in Table 2, the aggregated of all risk parameters estimated by experts is represented in a triangular fuzzy number. For example, the aggregated TFNs of RL is $R L^{*}=(0.75,0.33)$ and the corresponding fuzzy sets $R L^{*}$ is obtained by taking the intersection between TFN and the fuzzy term sets of $R L^{*}$ : 
TABLE 2: Experts' rating and its underlying fuzzy sets.

\begin{tabular}{|c|c|c|c|c|c|}
\hline \multirow{3}{*}{ Project management risk } & \multirow{3}{*}{ Experts } & \multicolumn{4}{|c|}{ Evaluation } \\
\hline & & \multicolumn{2}{|r|}{$\mathrm{RL}$} & \multicolumn{2}{|r|}{ RS } \\
\hline & & Crisp & Fuzzy set & Crisp & Fuzzy set \\
\hline \multirow{4}{*}{ Processes } & E1 & 8 & $\{\mathrm{P}, 0.5\},\{\mathrm{F}, 0.33\}$ & 8 & $\{\mathrm{C}, 0.5\},\{\mathrm{Ca}, 0.33\}$ \\
\hline & E2 & 8 & $\{\mathrm{P}, 0.5\},\{\mathrm{F}, 0.33\}$ & 8 & $\{\mathrm{C}, 0.5\},\{\mathrm{Ca}, 0.33\}$ \\
\hline & E3 & 7 & $\{P, 1\}$ & 7 & $\{\mathrm{C}, 1\}$ \\
\hline & E4 & 7 & $\{\mathrm{P}, 1\}$ & 8 & $\{\mathrm{C}, 0.5\},\{\mathrm{Ca}, 0.33\}$ \\
\hline \multirow{4}{*}{ Resources } & E1 & 7 & $\{\mathrm{P}, 1\}$ & 7 & $\{\mathrm{C}, 1\}$ \\
\hline & E2 & 6 & $\{\mathrm{O}, 0.5\},\{\mathrm{P}, 0.5\}$ & 7 & $\{\mathrm{C}, 1\}$ \\
\hline & E2 & 6 & $\{\mathrm{O}, 0.5\},\{\mathrm{P}, 0.5\}$ & 6 & $\{\mathrm{~S}, 0.5\},\{\mathrm{C}, 0.5\}$ \\
\hline & E4 & 5 & $\{\mathrm{O}, 1\}$ & 7 & $\{C, 1\}$ \\
\hline \multirow{4}{*}{ Inexperience team } & E1 & 8 & $\{P, 0.5\},\{F, 0.33\}$ & 6 & $\{\mathrm{~S}, 0.5\},\{\mathrm{C}, 0.5\}$ \\
\hline & E2 & 7 & $\{\mathrm{P}, 1\}$ & 8 & $\{\mathrm{C}, 0.5\},\{\mathrm{Ca}, 0.33\}$ \\
\hline & E2 & 5 & $\{\mathrm{O}, 1\}$ & 7 & $\{\mathrm{C}, 1\}$ \\
\hline & E4 & 6 & $\{\mathrm{O}, 0.5\},\{\mathrm{P}, 0.5\}$ & 8 & $\{\mathrm{C}, 0.5\},\{\mathrm{Ca}, 0.33\}$ \\
\hline \multirow{4}{*}{ Lack of management/control } & E1 & 7 & $\{\mathrm{P}, 1\}$ & 7 & $\{\mathrm{C}, 1\}$ \\
\hline & E2 & 6 & $\{\mathrm{O}, 0.5\},\{\mathrm{P}, 0.5\}$ & 8 & $\{\mathrm{C}, 0.5\},\{\mathrm{Ca}, 0.33\}$ \\
\hline & E2 & 5 & $\{\mathrm{O}, 1\}$ & 7 & $\{\mathrm{C}, 1\}$ \\
\hline & E4 & 6 & $\{\mathrm{O}, 0.5\},\{\mathrm{P}, 0.5\}$ & 6 & $\{\mathrm{~S}, 0.5\},\{\mathrm{C}, 0.5\}$ \\
\hline
\end{tabular}

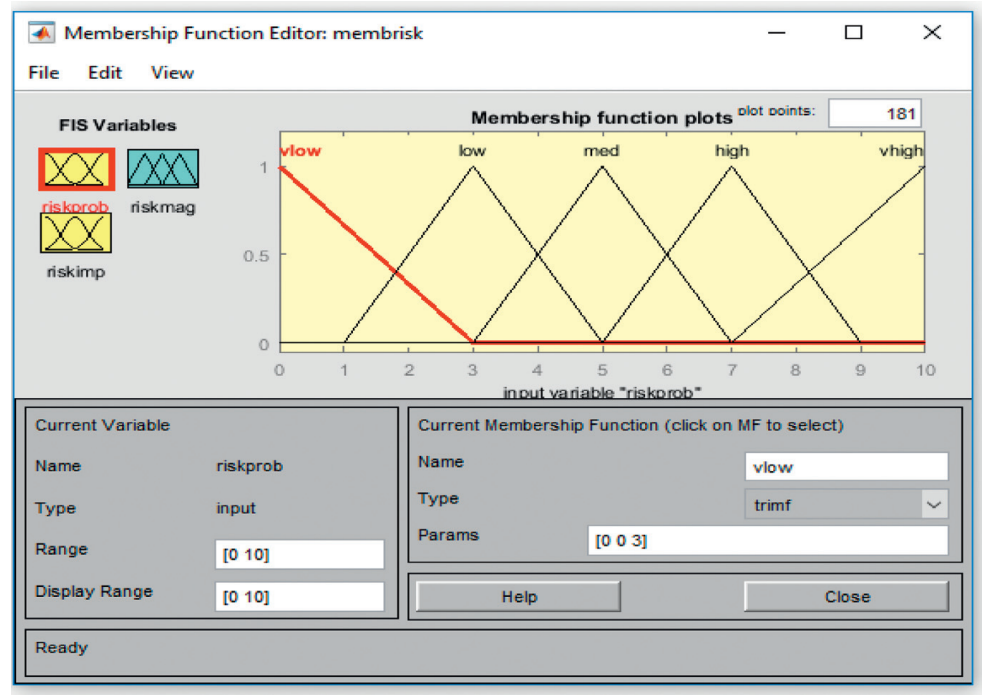

FIgURE 4: The attributes and membership functions of the input variable.

$R L^{\hat{*}}=\{($ Probable, 0.75, Frequent, 0.33$)\}$.

Likewise, we obtained the matching fuzzy sets of risk severity:

$$
R S^{\hat{*}}=\{(\text { Critical, 0.63), (Catasthropic, } 0.33)\} .
$$

Then, we evaluated the fuzzy design rules. The calculation of the fuzzy aggregation operator is displayed in Table 3. However, the final output of the risk criteria must be crisp numbers to be used in the risk assessment.

\subsection{Fuzzy Inference}

6.3.1. If-Then Rules Are the Basis of Fuzzy Conclusions. Since each of the two input factors (RL, RS) has one from the five linguistic variables (i.e., Improbable, Remote,
Occasional, Probable, or Frequent), the total number of combinations is $25(5 \times 5)$, this is the number of rules within this research. Table 4 lists some of the fuzzy if-then rules used in this research.

These rules are interpreted as follows:

Rule 1: if RL is IMPROBABLE and RS is NEGLIGIBLE, then the RC is TOLERABLE

Rule 2: if RL is IMPROBABLE and RS is SERIOUS, then the $\mathrm{RC}$ is LOW

From here, the pattern of the rules continues.

The fuzzy rule-based modelling is important particularly where the relations between the components of the system are not exactly known. If there are insufficient data for the analysis and if the data are uncertain about a particular thing which the user needs [32], rule implications of min-max are 
TABle 3: Fuzzy aggregation.

\begin{tabular}{|c|c|c|}
\hline Item & Criteria & Fuzzy aggregation \\
\hline \multirow{4}{*}{ Processes } & $R L$ & $\begin{array}{c}P: \mu=0.5+0.5+1+1 / 4=0.75 \\
F: \mu=0.33+0.33 / 2=0.33\end{array}$ \\
\hline & $R S$ & $\begin{array}{c}C: \mu=0.5+0.5+1+0.5 / 4=0.63 \\
C a: \mu=0.33+0.33+0.33 / 3=0.33\end{array}$ \\
\hline & $R L^{*}$ & $\{(\mathrm{P}, 0.75),(\mathrm{F}, 0.33)\}$ \\
\hline & $R S^{*}$ & $\{(\mathrm{C}, 0.63),(\mathrm{Ca}, 0.33)\}$ \\
\hline \multirow{5}{*}{ Resources } & $R L$ & $O: \mu=0.67+0.67 / 2=0.67$ \\
\hline & & $\begin{array}{c}P: \mu=0.67+0.67 / 2=0.67 \\
S: \mu=0.5+0.5 / 2=0.5\end{array}$ \\
\hline & $R S$ & $C: \mu=0.88+0.88 / 2=0.88$ \\
\hline & $R L^{*}$ & $\{(\mathrm{O}, 0.67),(\mathrm{P}, 0.67)\}$ \\
\hline & $R S^{*}$ & $\{(\mathrm{~S}, 0.5),(\mathrm{C}, 0.88)\}$ \\
\hline \multirow{8}{*}{ Inexperience team } & & $O: \mu=0.75+0.75+0.75 / 3=0.75$ \\
\hline & $R L$ & $P: \mu=0.63+0.63+0.63 / 3=0.63$ \\
\hline & & $F: \mu=0.33$ \\
\hline & & $S: \mu=0.5$ \\
\hline & RS & $C: \mu=0.63$ \\
\hline & & $\mathrm{Ca}: \mu=0.33+0.33 / 2=0.33$ \\
\hline & $R L^{*}$ & $\{(\mathrm{O}, 0.75),(\mathrm{P}, 0.61),(\mathrm{F}, 0.33)\}$ \\
\hline & $R S^{*}$ & $\{(\mathrm{~S}, 0.5),(\mathrm{C}, 0.583),(\mathrm{Ca}, 0.33)\}$ \\
\hline \multirow{7}{*}{ Lack of management/control } & & $O: \mu=0.67$ \\
\hline & $\mathrm{RL}$ & $P: \mu=0.67$ \\
\hline & & $S: \mu=0.5$ \\
\hline & RS & $C: \mu=0.75$ \\
\hline & & $\mathrm{Ca}: \mu=0.33$ \\
\hline & $R L^{*}$ & $\{(\mathrm{O}, 0.67),(\mathrm{P}, 0.67)\}$ \\
\hline & $R S^{*}$ & $\{(\mathrm{~S}, 0.5),(\mathrm{C}, 0.75),(\mathrm{Ca}, 0.33\}$ \\
\hline
\end{tabular}

TABLE 4: If-then rules.

\begin{tabular}{ccccccc}
\hline \multirow{2}{*}{ Risk criteria } & & & \multicolumn{3}{c}{ Risk likelihood } \\
& & $\mathrm{I}$ & $\mathrm{R}$ & $\mathrm{O}$ & $\mathrm{P}$ & Low \\
\hline & $\mathrm{N}$ & Tolerable & Tolerable & Low & Medium & Medium \\
Risk severity & $\mathrm{Mi}$ & Tolerable & Low & Low & High & High \\
& $\mathrm{S}$ & Low & Low & High & High \\
& $\mathrm{C}$ & Low & Medium & High & High & Intolerable \\
\hline
\end{tabular}

Four experts in the risk assessment group were asked to provide their assessment of the $R L$ and $R S$ in a predetermined scoring system, which is $0-10$.

used in this example. The 23 if-then rules that provide a more tangible understanding of the constructed system are depicted in Table 5. Generally, the fuzzy inference is divided into four steps as follows:

(a) Identify the if-then rules as in Table 5

(b) The minimum operator shall be taken into account to determine the strength of the rules and the calculations are in Table 6 (c) Control outputs: the result of the antecedent evaluation is applied to the membership function by "clipping" the consequent MF to the level of the truth value of the antecedent rule

(d) Consider the operator to calculate the maximum output of the fuzzy decision

The output variable is the overall risk criteria of the project, which has five linguistic variables. The degree of membership functions is given by the equation below:

$$
\mu_{\mathrm{agg}}\left(R C^{*}\right) \text { process }=\max \left\{\min \left(0.63, \mu H\left(R C^{*}\right)\right), \min \left(0.33, \mu I N\left(R C^{*}\right)\right)\right\} .
$$


Table 5: Mapping of $R L^{*} \times R S^{*}$ (list of fuzzy if-then rule).

\begin{tabular}{|c|c|}
\hline Item & If-Then rules \\
\hline$r 1$ & $\begin{array}{l}\text { If } \mathrm{RL} \text { is probable and the } \mathrm{RS} \text { is critical, then } \mathrm{RC} \text { is high } \\
\text { If } \mathrm{RL} \text { is probable and the } \mathrm{RS} \text { is catastrophic, then } \mathrm{RC} \text { is high } \\
\text { If } \mathrm{RL} \text { is frequent and the RS is critical, then } \mathrm{RC} \text { is high } \\
\text { If } \mathrm{RL} \text { is frequent and the RS is catastrophic, then } \mathrm{RC} \text { is intolerable }\end{array}$ \\
\hline$r 2$ & $\begin{array}{l}\text { If RL is occasional and RS is serious, then RC is medium } \\
\text { If RL is occasional and RS is critical, then RC is high } \\
\text { If RL is probable and RS is serious, then RC is high } \\
\text { If RL is probable and RS is critical, then RC is high }\end{array}$ \\
\hline$r 3$ & $\begin{array}{l}\text { If RL is occasional and RS is serious, then RC is medium } \\
\text { If RL is occasional and RS is critical, then RC is high } \\
\text { If RL is occasional and RS is catastrophic, then RC is high } \\
\text { If RL is probable and RS is serious, then RC is high } \\
\text { If RL is probable and RS is critical, then RC is high } \\
\text { If RL is probable and RS catastrophic, then RC is high } \\
\text { If RL is frequent and RS is serious, then RC is high } \\
\text { If RL is frequent and RS is critical, then RC is high } \\
\text { If RL is frequent and RS is catastrophic, then RC is intolerable }\end{array}$ \\
\hline$r 4$ & $\begin{array}{l}\text { If } \mathrm{RL} \text { is occasional and RS is serious, then } \mathrm{RC} \text { is medium } \\
\text { If } \mathrm{RL} \text { is occasional and RS is critical, then RC is high } \\
\text { If } \mathrm{RL} \text { is occasional and RS is catastrophic, then RC is high } \\
\text { If RL is probable and RS is serious, then RC is high } \\
\text { If RL is probable and RS is critical, then RC is high } \\
\text { If RL is probable and RS is catastrophic, then RC is high }\end{array}$ \\
\hline
\end{tabular}

TABLe 6: Minimum operator.

\begin{tabular}{|c|c|}
\hline Item & Minimum operator \\
\hline$r 1$ & $\begin{array}{l}\mathrm{R} 1: \alpha_{1}=\mu P\left(R L^{*}\right) \hat{\mu} C\left(R S^{*}\right)=\min (0.75,0.625)=0.63 \\
\mathrm{R} 2: \alpha_{2}=\mu P\left(R L^{*}\right) \hat{\mu} C a\left(R S^{*}\right)=\min (0.75,0.33)=0.33 \\
\mathrm{R} 3: \alpha_{3}=\mu F\left(R L^{*}\right) \hat{\mu} C\left(R S^{*}\right)=\min (0.33,0.625)=0.33 \\
\mathrm{R} 4: \alpha_{4}=\mu F\left(R L^{*}\right) \hat{\mu} C a\left(R S^{*}\right)=\min (0.33,0.33)=0.33\end{array}$ \\
\hline$r 2$ & $\begin{array}{c}\mathrm{R} 1: \alpha_{1}=\mu O\left(R L^{*}\right) \hat{\mu} S\left(R S^{*}\right)=\min (0.67,0.5)=0.5 \\
\mathrm{R} 2: \alpha_{2}=\mu O\left(R L^{*}\right) \hat{\mu} C\left(R S^{*}\right)=\min (0.67,0.88)=0.67 \\
\mathrm{R} 3: \alpha_{3}=\mu P\left(R L^{*}\right) \hat{\mu} S\left(R S^{*}\right)=\min (0.67,0.5)=0.5 \\
\mathrm{R} 4: \alpha_{4}=\mu P\left(R L^{*}\right) \hat{\mu} C\left(R S^{*}\right)=\min (0.67,0.88)=0.67\end{array}$ \\
\hline$r 3$ & $\begin{array}{c}\text { R1: } \alpha_{1}=\mu O\left(R L^{*}\right) \hat{\mu} S\left(R S^{*}\right)=\min (0.75,0.5)=0.5 \\
\text { R2: } \alpha_{2}=\mu O\left(R L^{*}\right) \hat{\mu} C\left(R S^{*}\right)=\min (0.75,0.63)=0.63 \\
\text { R3: } \alpha_{3}=\mu O\left(R L^{*}\right) \hat{\mu} C a\left(R S^{*}\right)=\min (0.75,0.33)=0.33 \\
\text { R4: } \alpha_{4}=\mu P\left(R L^{*}\right) \hat{\mu} S\left(R S^{*}\right)=\min (0.67,0.5)=0.5 \\
\text { R5: } \alpha_{5}=\mu P\left(R L^{*}\right) \hat{\mu} C\left(R S^{*}\right)=\min (0.67,0.63)=0.63 \\
\text { R6: } \alpha_{6}=\mu P\left(R L^{*}\right) \hat{\mu} C a\left(R S^{*}\right)=\min (0.67,0.33)=0.33 o \\
\text { R7: } \alpha_{7}=\mu F\left(R L^{*}\right) \hat{\mu} S\left(R S^{*}\right)=\min (0.33,0.5)=0.33 \\
\text { R8: } \alpha_{8}=\mu F\left(R L^{*}\right) \hat{\mu} C\left(R S^{*}\right)=\min (0.33,0.63)=0.33 \\
\text { R9: } \alpha_{9}=\mu F\left(R L^{*}\right) \hat{\mu} C a\left(R S^{*}\right)=\min (0.33,0.33)=0.33\end{array}$ \\
\hline$r 4$ & $\begin{array}{c}\mathrm{R} 1: \alpha_{1}=\mu O\left(R L^{*}\right) \hat{\mu} S\left(R S^{*}\right)=\min (0.67,0.5)=0.5 \\
\mathrm{R} 2: \alpha_{2}=\mu O\left(R L^{*}\right) \hat{\mu} C\left(R S^{*}\right)=\min (0.67,0.75)=0.67 \\
\mathrm{R} 3: \alpha_{3}=\mu O\left(R L^{*}\right) \hat{\mu} C a\left(R S^{*}\right)=\min (0.67,0.33)=0.33 \\
\mathrm{R} 4: \alpha_{4}=\mu P\left(R L^{*}\right) \hat{\mu} S\left(R S^{*}\right)=\min (0.67,0.5)=0.5 \\
\mathrm{R} 5: \alpha_{5}=\mu P\left(R L^{*}\right) \hat{\mu} C\left(R S^{*}\right)=\min (0.67,0.75)=0.75 \\
\mathrm{R} 1: \alpha_{1}=\mu P\left(R L^{*}\right) \hat{\mu} C\left(R S^{*}\right)=\min (0.75,0.625)=0.63\end{array}$ \\
\hline
\end{tabular}


TABle 7: Control outputs.

\begin{tabular}{|c|c|c|}
\hline Item & Minimum operator & Description \\
\hline Processes & $\begin{aligned} \mathrm{R} 1: \alpha_{1} \hat{H}\left(R C^{*}\right) & =\min \left(0.63, \mu H\left(R C^{*}\right)\right) \\
\mathrm{R} 2: \alpha_{2} \hat{H}\left(R C^{*}\right) & =\min \left(0.33, \mu H\left(R C^{*}\right)\right) \\
\mathrm{R} 3: \alpha_{3} \hat{H}\left(R C^{*}\right) & =\min \left(0.33, \mu H\left(R C^{*}\right)\right) \\
\mathrm{R} 4: \alpha_{4} \hat{I} N\left(R C^{*}\right) & =\min \left(0.33, \mu I N\left(R C^{*}\right)\right)\end{aligned}$ & $\begin{array}{c}\operatorname{Max}(\text { high })=0.63 \\
\operatorname{Max}(I N)=0.33\end{array}$ \\
\hline Resources & $\begin{array}{l}\mathrm{R} 1: \alpha_{1} \hat{M}\left(R C^{*}\right)=\min \left(0.5, \mu M\left(R C^{*}\right)\right) \\
\mathrm{R} 2: \alpha_{2} \hat{H}\left(R C^{*}\right)=\min \left(0.67, \mu H\left(R C^{*}\right)\right) \\
\mathrm{R} 3: \alpha_{3} \hat{H}\left(R C^{*}\right)=\min \left(0.5, \mu H\left(R C^{*}\right)\right) \\
\mathrm{R} 4: \alpha_{4} \hat{H}\left(R C^{*}\right)=\min \left(0.553, \mu H\left(R C^{*}\right)\right)\end{array}$ & $\begin{array}{c}\operatorname{Max}(\text { medium })=0.5 \\
\operatorname{Max}(\text { high })=0.67\end{array}$ \\
\hline Inexperience team & $\begin{array}{l}\text { R1: } \alpha_{1} \hat{M}\left(R C^{*}\right)=\min \left(0.5, \mu M\left(R C^{*}\right)\right) \\
\text { R2: } \alpha_{2} \hat{H}\left(R C^{*}\right)=\min \left(0.583, \mu H\left(R C^{*}\right)\right) \\
\text { R3: } \alpha_{3} \hat{H}\left(R C^{*}\right)=\min \left(0.33, \mu H\left(R C^{*}\right)\right) \\
\text { R4: } \alpha_{4} \hat{H}\left(R C^{*}\right)=\min \left(0.5, \mu H\left(R C^{*}\right)\right) \\
\text { R5: } \alpha_{5} \hat{H}\left(R C^{*}\right)=\min \left(0.583, \mu H\left(R C^{*}\right)\right) \\
\text { R6: } \alpha_{6} \hat{H}\left(R C^{*}\right)=\min \left(0.33, \mu H\left(R C^{*}\right)\right) \\
\text { R7: } \alpha_{7} \hat{H}\left(R C^{*}\right)=\min \left(0.33, \mu H\left(R C^{*}\right)\right) \\
\text { R8: } \alpha_{8} \hat{H}\left(R C^{*}\right)=\min \left(0.33, \mu H\left(R C^{*}\right)\right) \\
\text { R9: } \alpha_{9} \hat{I} N\left(R C^{*}\right)=\min \left(0.33, \mu I N\left(R C^{*}\right)\right)\end{array}$ & $\begin{array}{c}\operatorname{Max}(\text { medium })=0.5 \\
\operatorname{Max}(\text { high })=0.583 \\
\operatorname{Max}(\text { IN })=0.33\end{array}$ \\
\hline Lack of management/control & $\begin{array}{l}\text { R1: } \alpha_{1} \hat{M}\left(R C^{*}\right)=\min \left(0.5, \mu M\left(R C^{*}\right)\right) \\
\text { R2: } \alpha_{2} \hat{H}\left(R C^{*}\right)=\min \left(0.75, \mu H\left(R C^{*}\right)\right) \\
\text { R3: } \alpha_{3} \hat{H}\left(R C^{*}\right)=\min \left(0.33, \mu H\left(R C^{*}\right)\right) \\
\text { R4: } \alpha_{4} \hat{H}\left(R C^{*}\right)=\min \left(0.5, \mu H\left(R C^{*}\right)\right) \\
\text { R5: } \alpha_{5} \hat{H}\left(R C^{*}\right)=\min \left(0.553, \mu H\left(R C^{*}\right)\right) \\
\text { R6: } \alpha_{6} \hat{H}\left(R C^{*}\right)=\min \left(0.33, \mu H\left(R C^{*}\right)\right)\end{array}$ & $\begin{array}{c}\operatorname{Max}(\text { medium })=0.5 \\
\operatorname{Max}(\text { high })=0.75\end{array}$ \\
\hline
\end{tabular}

6.4. Defuzzification. The degree of membership of the conclusion is sometimes interpreted as the "degree of truth" $[32,33]$. In the preceding example, the support value at the maximal degree of membership and the truth value of each fuzzy conclusion (see Tables 1 and 7) are the following. For example, refer to the risk on the "inexperience project team." The maximum support value and truth value (degree of membership) of the medium level of risk are 5 and $0.5(\mu$ $($ medium risk $)=\max (0,0.5)=0.5)$, respectively. The maximum support value and truth value (degree of membership) of high risk are 7 and $0.583(\mu$ (high risk) $=\max (0.583,0.33$, $0.5,0.583,0.33,0.33,0.33)=0.583)$, respectively. The maximum support value and truth value (degree of membership) of intolerable risk are 10 and $0.33(\mu($ IN risk $)=0.33)$, respectively. Hence, applying to (7), the $Z$ value for all the risk items is shown in Table 8.

Using a numerical example through the illustration of risk criteria, a comparison of the results using matrix likelihood-severity (LS) and traditional method is presented in Table 9. The table summarizes that the risk yields similarities between fuzzy decision and traditional aggregating experts' estimates. The apparent disadvantage of the traditional method is that different sets of RL and RS may generate an identical value of risk; however, the risk criteria (Level) may not necessarily be the same in the real term. For example, two risks, namely, process $(r 1)$ and inexperience team $(r 3)$ estimated by the experts will give a value of 7 and 8 , respectively. The scoring value for risk level yields the same index which is 56 (risk between medium to high). However, in real problem, the risk criteria of these two may vary. The advantage of using Mamdani-type in assessing project risk is that it captures uncertainty in the multiexpert's inputs despite providing richer information on the particular risk. The traditional risk method does not take into account the relative value of RL and RS, so this may not be accurate in real problems. 


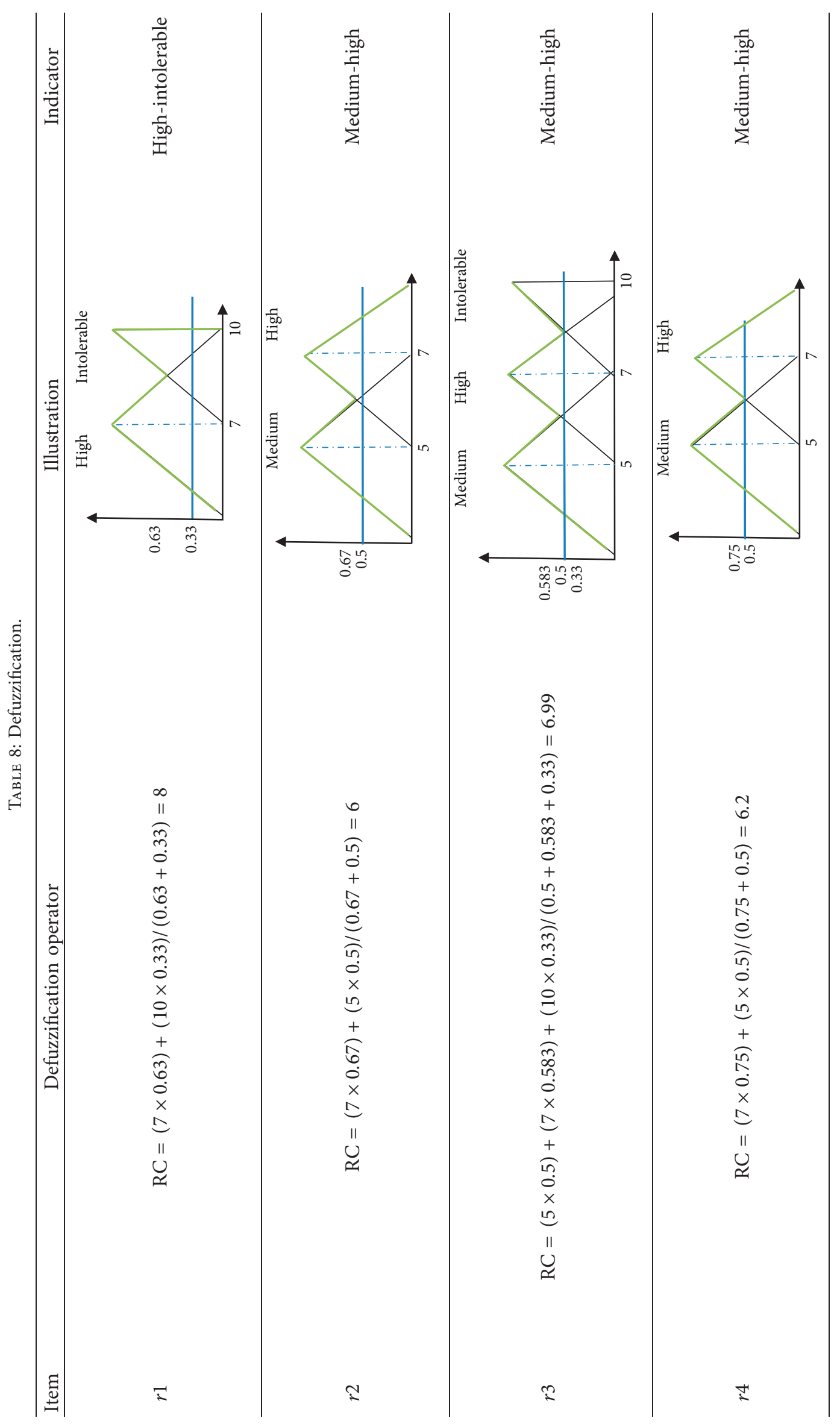


TABLE 9: Comparison of risk assessment.

\begin{tabular}{|c|c|c|c|c|c|c|c|c|}
\hline \multirow{3}{*}{ Risk } & \multicolumn{4}{|c|}{ Input risk parameter } & \multicolumn{4}{|c|}{ Output } \\
\hline & \multicolumn{2}{|c|}{ Crisp } & \multicolumn{2}{|c|}{ Fuzzy } & \multicolumn{2}{|c|}{ Traditional } & \multicolumn{2}{|c|}{ Fuzzy } \\
\hline & $\mathrm{RL}$ & RS & RL & RS & $\mathrm{RC}$ & Rank & $\mathrm{RC}$ & Rank \\
\hline$r 1$ & 7.5 & 7.75 & $\{(\mathrm{P}, 0.75),(\mathrm{F}, 0.33)\}$ & $(\mathrm{C}, 0.63),(\mathrm{Ca}, 0.33)$ & 58.13 & 1 & 8 & 1 \\
\hline$r 2$ & 6 & 6.75 & $(\mathrm{O}, 0.67),(\mathrm{P}, 0.67)$ & $(\mathrm{S}, 0.5),(\mathrm{C}, 0.88)$ & 40.5 & 4 & 6 & 4 \\
\hline$r 3$ & 6.5 & 7.25 & $(\mathrm{O}, 0.75),(\mathrm{P}, 0.61),(\mathrm{F}, 0.33)$ & $(\mathrm{S}, 0.5),(\mathrm{C}, 0.583),(\mathrm{Ca}, 0.33)$ & 47.13 & 2 & 6.99 & 2 \\
\hline$r 4$ & 6 & 7 & $(\mathrm{O}, 0.67),(\mathrm{P}, 0.67)$ & $(\mathrm{S}, 0.5),(\mathrm{C}, 0.75),(\mathrm{Ca}, 0.33$ & 42 & 3 & 6.2 & 3 \\
\hline
\end{tabular}

\section{Conclusions and Future Work}

The use of fuzzy inference system is an added advantage for users throughout complicated risk assessments especially when suitable data for assessing risk are not assessable. The use of Mamdani-type fuzzy inference process in risk assessment integrates both qualitative and quantitative approaches. This can be seen that misguided decisions will cause project failure especially in Malaysia construction industry where the risk management practice is still in its infancy. Decision-making assistance does not alleviate the decision-maker's responsibility; however, it offers useful knowledge and thereby increases the efficiency of decisionmaking in the risk assessment process. In addition, there are other advantages to the proposed system, namely, the relationship between inputs and outputs was linguistic. The model has been made more flexible to adapt under varying circumstances, and the decision-making process has been made easier and quicker. As the findings were more comprehensible and credible, the proposed risk assessment methodology entails greater advantage especially when fuzzy and/or crisps' preferences are presented. Furthermore, it can be concluded that the fuzzy mathematical approach reduces uncertainty and improves accuracy which is ideal for decision maker in complicated risk assessments due to its model flexibility. It is therefore a very effective tool when appropriate risk assessment data are not accessible.

\section{Data Availability}

No data were used to support this study.

\section{Conflicts of Interest}

The authors declare that they have no conflicts of interest.

\section{Acknowledgments}

The authors are very thankful to the management team of the project constructions based in Malaysia for their inputs.

\section{References}

[1] V. K. Gupta and J. J. Thakkar, "A quantitative risk assessment methodology for construction project," Sadhana-Academy Proceedings in Engineering Sciences, vol. 43, no. 7, pp. 1-16, 2018.

[2] W. Hürlimann, R. Agliardi, P. Popivanov et al., N. Banaitiene, Risk Management-Current Issues and Challenges, IntechOpen, London, UK, 2012.
[3] P. X. W. Zou, G. Zhang, and J. Wang, "Understanding the key risks in construction projects in China," International Journal of Project Management, vol. 25, no. 6, pp. 601-614, 2007.

[4] R. Doskočil, "An evaluation of total project risk based on fuzzy logic," Business: Theory and Practice, vol. 15, no. 2, pp. 23-31, 2016.

[5] H. Abd El Khalek, "Risk and uncertainty assessment model in construction projects using fuzzy logic," American Journal of Civil Engineering, vol. 4, no. 1, p. 24, 2016.

[6] S. Khaleghi, S. Givehchi, and S. Karimi, "Fuzzy risk assessment and categorization, based on event tree analysis (ETA) and layer of protection analysis (LOPA): case study in gas transport system," World Applied Programming, vol. 3, no. 9, pp. 417-426, 2013.

[7] B. G. Kang, M. A. Fazlie, B. H. Goh, M. K. Song, and C. Zhang, "Current practice of risk management in the Malaysia construction industry-the process and tools/techniques," International Journal of Structural and Civil Engineering Research, vol. 4, no. 4, pp. 371-377, 2015.

[8] L. C. Siang and A. S. Ali, "Implementation of risk management in the Malaysian construction industry," Journal of Surveying, Construction \& Property, vol. 3, no. 1, pp. 1-15, 2019.

[9] J. Zeng, M. An, and A. H. C. Chan, “A fuzzy reasoning decision making approach based multi-expert judgement for construction project risk analysis," Association of Researchers in Construction Management, ARCOM 2005-Proceedings of the 21st Annual Conference, vol. 2, no. September, pp. 841852, 2005.

[10] B. Mondal, D. Mazumdar, and S. Raha, "Similarity in approximate reasoning," International Journal of Computational Cognition, vol. 4, no. 3, pp. 46-56, 2006.

[11] R. Ketata, H. Bellaaj, M. Chtourou, and M. B. Amer, “Adjustment of membership functions, generation and reduction of fuzzy rule base from numerical data," Malaysian Journal of Computer Science, vol. 20, no. 2, pp. 147-169, 2017.

[12] D. Forbes, S. Smith, and M. Horner, "Tools for selecting appropriate risk management techniques in the built environment," Construction Management and Economics, vol. 26, no. 11, pp. 1241-1250, 2008.

[13] Q. Meng, X. Qu, X. Wang, V. Yuanita, and S. C. Wong, "Quantitative risk assessment modeling for nonhomogeneous urban road tunnels," Risk Analysis, vol. 31, no. 3, pp. 382-403, 2010.

[14] D. Mercurio, L. Podofillini, E. Zio, and V. N. Dang, "Identification and classification of dynamic event tree scenarios via possibilistic clustering: application to a steam generator tube rupture event," Accident Analysis \& Prevention, vol. 41, no. 6, pp. 1180-1191, 2009.

[15] R. Olawoyin, "Risk and reliability evaluation of gas connector systems using fuzzy theory and expert elicitation," Cogent Engineering, vol. 4, no. 1, 2017. 
[16] M. d. M. Paranhos, S. J. Bachega, D. M. Tavares, and N. F. S. Calife, "Aplicação da análise de modo e efeitos de falha para o gerenciamento de riscos de um projeto," Sistemas \& Gestão, vol. 11, no. 4, pp. 444-454, 2017.

[17] A. Dziadosz and M. Rejment, "Risk analysis in construction project - chosen methods," Procedia Engineering, vol. 122, no. January 2016, pp. 258-265, 2015.

[18] A. Yazdani, S. Shariati, and A. Yazdani-Chamzini, "A risk assessment model based on fuzzy logic for electricity distribution system asset management," Decision Science Letters, vol. 3, no. 3, pp. 343-352, 2014.

[19] J.-M. Kim, T. Kim, K. Son, J. Bae, and S. Son, “A quantitative risk assessment development using risk indicators for predicting economic damages in construction sites of South Korea," Journal of Asian Architecture and Building Engineering, vol. 18, no. 5, pp. 472-478, 2019.

[20] B. Barghi and S. Shadrokh sikari, "Qualitative and quantitative project risk assessment using a hybrid PMBOK model developed under uncertainty conditions," Heliyon, vol. 6, no. 1, Article ID e03097, 2020.

[21] L. A. Zadeh, "The concept of a linguistic variable and its application to approximate reasoning-I," Information Sciences, vol. 8, no. 3, pp. 199-249, 1975.

[22] L. A. Zadeh, "Fuzzy sets *," Information and Control, vol. 353, no. 8, pp. 338-353, 1965.

[23] M. R. Civanlar and H. J. Trussell, "Constructing membership functions using statistical data," Fuzzy Sets and Systems, vol. 18, no. 1, pp. 1-13, 1986.

[24] J. Zeng, M. An, A. Chan, and Y. Lin, "A methodology for assessing risks in the construction process," Proceedings of the Twentieth Annual, vol. 2, no. September, pp. 1-3, 2004.

[25] E. H. Mamdani and S. Assilian, "An experiment in linguistic synthesis with a fuzzy logic controller," International Journal of Man-Machine Studies, vol. 7, no. 1, pp. 1-13, 2017.

[26] T. Takagi and M. Sugeno, "Fuzzy identification of systems and its application to modeling and control," IEEE Transactions on Systems, Man, and Cybernetics, vol. 15, pp. 116-132, 2017.

[27] B. Bouchon-Meunier, M. Dotoli, and B. Maione, "On the choice of membership functions in a mamdani-type fuzzy controller," in Proceedings of the First Online Workshop on Soft Computing, pp. 1-6, August 1996.

[28] A. Kaur and A. Kaur, "Comparison of mamdani-type and sugeno-type fuzzy inference systems for air conditioning system," International Journal of Soft Computing \& Engineering, vol. 2, no. 2, pp. 323-325, 2012.

[29] C. M. Feng and C. C. Chung, "Assessing the risks of airport airside through the fuzzy logic-based failure modes, effect, and criticality analysis," Mathematical Problems in Engineering, vol. 2013, Article ID 239523, 11 pages, 2013.

[30] A. Nieto-Morote and F. Ruz-Vila, "A fuzzy approach to construction project risk assessment," International Journal of Project Management, vol. 29, no. 2, pp. 220-231, 2011.

[31] V. Carr and J. H. Tah, "A fuzzy approach to construction project risk assessment and analysis: construction project risk management system," Advances in Engineering Software, vol. 32, no. 10-11, pp. 847-857, 2017.

[32] K. Shang and Z. Hossen, Applying Fuzzy Logic to Risk Assessment and Decision-Making, pp. 1-59, Canadian Institute of Actuaries, Ottawa, Canada, 2013.

[33] T. Bilgiç and I. Türkşen, "Measurement of membership functions: theoretical and empirical work," Fundamentals of Fuzzy Sets, vol. 7, pp. 195-227, 2000. 